STONE DISEASE

doi: $10.1590 / \mathrm{S} 1677-553820100002000014$

\title{
Percutaneous nephrolithotomy for proximal ureteral calculi with severe hydronephrosis: assessment of different lithotriptors
}

Zhu Z, Xi Q, Wang S, Liu J, Ye Z, Yu X, Bai J, Li C

Department of Urology, Tongji Hospital, Tongji Medical College, Huazhong University of Science and Technology, Wuhan, China

J Endourol. 2010; 24: 201-5

Purpose: We compared the efficacy and safety of percutaneous nephrolithotomy (PCNL) with different intracorporeal lithotriptors for proximal ureteral stones in patients with severe hydronephrosis.

Patients and Methods: We retrospectively analyzed the records of 192 patients with proximal ureteral calculi and severe hydronephrosis who underwent PCNL between February 2003 and December 2007. Calculi were fragmented with a pneumatic lithotriptor in 44 patients (group 1), Swiss Lithoclast Master in 54 (group 2), low-power holmium:yttrium-aluminum-garnet (YAG) laser in 56 (group 3) and high-power holmium:YAG laser in 38 (group 4). Patients were assessed about 12 months postoperatively with intravenous urography and ultrasonography for late complications. Stone size, operative time, stone-free rate, and follow-up were analyzed in each group.

Results: Mean stone size for different groups were $16.2+/-2.8 \mathrm{~mm}, 16.6+/-2.1 \mathrm{~mm}, 16.0+/-2.7 \mathrm{~mm}$, and $16.4+/-1.1 \mathrm{~mm}$, respectively. Average operative time for different groups were $118+/-17$ minutes, $81+/-10$ minutes, $85+/-14$ minutes, $110+/-16$ minutes, respectively. Group 2 and group 3 showed superior outcomes of shorter operative time $(\mathrm{P}=0.000)$. The overall stone-free rate was $86.5 \%$. As stratified by lithotriptors, the stone-free rate was $81.8 \%$ in group $1,92.9 \%$ in group $2,88.9 \%$ in group 3 , and $78.9 \%$ in group $4(\mathrm{P}=0.190)$. No significant difference was found among the groups in terms of blood loss and postoperative hospital stay. Repeated PCNL or shockwave lithotripsy was necessary as an auxiliary procedure in 26 patients. The overall complication rate was $18.2 \%$; most complications were minor and insignificant. During the follow-up, ureteral stricture developed in 10 patients and new renal stones developed in 4 patients.

Conclusions: PCNL combined with Swiss Lithoclast Master or low-power holmium:YAG laser is the preferred endourologic modality for the management of proximal ureteral calculi in patients with severe hydronephrosis.

\section{Editorial Comment}

The authors do not comment on their use of basket devices for fragment removal. They comment that one deterrent to pneumatic lithotripsy is the large fragments that are formed, requiring "time-consuming" extraction. Indeed, the absence of a nephrostomy sheath in their surgical technique suggests that active fragment extraction is not performed. As such, this may impact their findings of higher efficacy for lithotripters that either form smaller fragments (low-energy HO:YAG) or actively extract stone fragments (ultrasonic component of the Lithoclast). The finding of a higher ureteral stricture rate with High-energy HO:YAG is an important contribution to the literature.

The authors do not report their selection of calyx for percutaneous access; one would anticipate a high percentage of upper and middle calyces to facilitate access to the proximal ureter with a rigid nephroscope. For centers selecting a lower calyceal access and the use of a flexible endoscope, the low-energy HO:YAG would come out on top.

Dr. Manoj Monga

Professor, Department of Urology

University of Minnesota

Edina, Minnesota, USA

E-mail:endourol@yahoo.com 